\title{
ADIABATIC SUPERNOVA EXPANSION INTO THE CIRCUMSTELLAR MEDIUM
}

\author{
DAVID L. BAND AND EDISON P. LIANG
}

Institute of Geophysics and Planetary Physics and Physics Department Lawrence Livermore National Laboratory, P.O. Box 808, Livermore, CA 94550

\begin{abstract}
We perform one dimensional numerical simulations with a Lagrangian hydrodynamics code of the adiabatic expansion of a supernova into the surrounding medium. The early expansion follows Chevalier's analytic selfsimilar solution until the reverse shock reaches the ejecta core. We follow the expansion as it evolves towards the adiabatic blast wave phase. Some memory of the earlier phases of expansion is retained in the interior even when the outer regions expand as a blast wave. We find the results are sensitive to the initial configuration of the ejecta and to the placement of gridpoints.
\end{abstract}

The surface of a young supernova (SN) does not expand freely until it sweeps up a sufficient mass of the surrounding material to form a blast wave. Due to the steep density gradient in the SN envelope, and a circumstellar medium (CSM) denser than the average interstellar medium (ISM), the density jump between the expanding ejecta and the surrounding medium may be relatively small, resulting in a decelerating interaction region long before a blast wave forms. The CSM may be the product of a slow, high density wind emitted by the progenitor for a short period before the explosion. An observable interaction region bounded by a forward and reverse shock can then form at the contact surface between the SN envelope and the surrounding medium. Most of the historical supernova remnants (SNRs) are in this pre-blast wave phase, and indeed some extragalactic $\mathrm{SNs}$ show radio emission from this early interaction (Weiler et al. 1986). Because this phase has observable consequences, and the relevant physical processes are not clear, we are undertaking a series of numerical studies that will trace the early evolution of a SN until a blast wave is formed. In the first study reported here we assume adiabatic hydrodynamic expansion.

The homologously expanding ejecta can be approximated as a constant density core surrounded by an envelope with a steep density gradient $\rho \propto r^{-n}$. The surrounding medium is either a constant density ISM, or a CSM with a $\rho \propto r^{-2}$ profile due to a constant velocity wind. Chevalier (1982a) developed a self-similar solution (SSS) for the expansion of an envelope with $\rho \propto r^{-n}, \quad n>5$, into a stationary region $\rho \propto r^{-8}$, assuming ideal hydrodynamics. This solution should be a valid approximation for the early SNR as long as the reverse shock remains within the envelope, and the forward shock remains within the CSM (if there is a CSM). After the SSS breaks down, the remnant eventually evolves into a Sedov-Taylor blast wave (which is also self-similar, but here will be referred to as the blast wave). The SSS reproduces observed radio light curves reasonably well with simple prescriptions for modeling the radio emission and absorption (Chevalier 1982b; Weiler et al. 1986). The transition between the SSS and the blast wave phases is of interest in modeling observed supernovae and understanding the evolution of the interstellar medium (Cioffi 1986, private communication). The transition phase cannot be studied analytically, and numerical calculations are required. In addition, the numerical calculations of Itoh and Fabian (1984) cast doubt on whether the SSS is reached before the conditions for its validity break down. Also, physical effects such as heat conduction, magnetic fields and inefficient coupling between electrons and ions may be relevant within the interaction region (Liang and Chevalier 1985).

The applicable physical processes are not clear. As a result of the low densities and the high temperatures, the Coulomb mean-free-path is comparable to the length scales of the interaction region. Thus, it would appear that hydrodynamics is a questionable assumption within the 
interaction region; thermal conduction is likely to be flux limited; and the shocks are collisionless. However, the electron gyroradius in the magnetic fields (whose presence is indicated by the radio synchrotron emission) is shorter than the scale of the SN, justifying the use of hydrodynamics (Chew, Goldberger, and Low 1956). Similarly, the magnetic fields should suppress thermal conduction across field lines, particularly since shock compression and spherical expansion will increase the ratio of the tangential to radial magnetic fields. Finally, observations of interplanetary shocks and numerical plasma simulations indicate that plasma processes nearly equilibrate the electron and ion temperatures in a collisionless shock. Consequently, even though the Coulomb mean-free-path is very long, ideal hydrodynamics is probably a good approximation of the applicable physics. In the first stage of our numerical studies we therefore assume ideal hydrodynamics; in future studies we will include the effects of flux-limited thermal conduction and different kinds of electron-ion thermal coupling.

The interaction region appears to be Rayleigh-Taylor unstable since the pressure and density gradients are opposite either in the shocked ejecta or the shocked CSM. The instability will be reduced if thermal conduction smooths out the temperature gradient. However, the magnetic fields will suppress thermal conduction; moreover, the magnetic fields should be amplified in the turbulence produced by the instabilities. This turbulence is beyond the capabilities of our current numerical methods, and consequently will not be included at this time.

The numerical calculations were performed on a Cray computer using an explicit Lagrangian finite difference scheme in which shocks are smoothed by von Neumann artificial viscosity. Typically energy is conserved to $1 \%$. We found that the spacing of the Lagrangian gridpoints is crucial to the success of the numerical calculations. The density varies by large factors over the various regions and the mass per zone must vary to provide the desired resolution. However, abrupt changes in the mass per zone introduce interfaces from which spurious shocks can be reflected. For example, the reflected shock found by Itoh and Fabian (1985) when the reverse shock reached the core-envelope interface is probably an artifact of the zoning; we found similar spurious reflections in test runs.

As an initial condition in our calculations, the power law envelope was extended to a radius close to the radius predicted by the SSS, and consequently the runs rapidly reached the SSS. In the calculations of Itoh and Fabian (1984) the SN did not expand with the predicted power law behavior because they truncated the envelopes at a much smaller radius than the predicted postion of the SSS contact surface. The density jump at their initial contact surface was very large, and thus the ejecta expanded at a nearly constant velocity. Indeed, in their runs with a CSM the contact surface expanding at its initial velocity would have intersected the predicted SSS contact surface only near the outer edge of the CSM. Thus the relevance of the SSS to actual SN expansion depends upon the radius to which the envelope extends.

We considered three different models. In all cases the explosion released an energy of $10^{51}$ ergs and the star was surrounded by an ISM density of $10^{-24} \mathrm{gm}-\mathrm{cm}^{-3}$. When there was a CSM interposed between the ejecta and the ISM, it had a mass of $1 M_{\odot}$ and had been emitted for $10^{4}$ years at a wind velocity of $10 \mathrm{~km}-\mathrm{sec}^{-1}$. Models of Type I SNs, with an ejecta mass of 1.4 $M_{\odot}$ and a $\rho \propto r^{-7}$ envelope, were allowed to expand into two types of media: a CSM followed by an ISM (the CSM case); and into an ISM alone (the ISM case). The model of a Type II SN, with a $10 M_{\odot}$ ejecta with a $\rho \propto r^{-12}$ envelope, was only expanded into a CSM followed by an ISM. The Type II SN calculation was qualitatively similar to the Type I SN calculation with a CSM, hence we will concentrate here on the two Type I calculations.

The position of the forward shock for the two Type I SN cases is shown on Figure 1. The initial SSS phase and final blast wave phase are both self-similar; for self-similar expansion the position of the forward shock is $r_{f \circ} \propto t^{\eta}$ (note $v_{f}=\eta r_{f} / t$ ). The effective power law index 


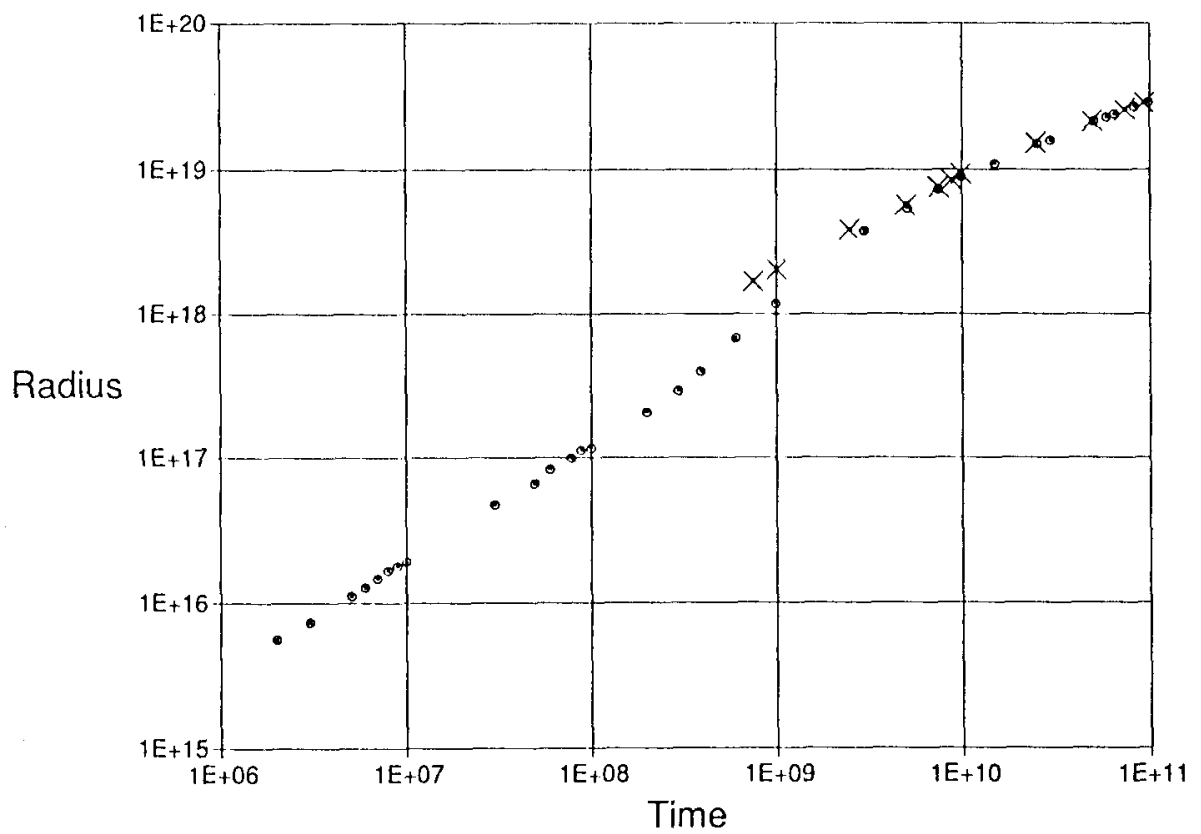

Figure 1-Position of Forward Shock. The expansion of a $1.4 M_{\odot}$ star with a $\rho \propto$ $r^{-7}$ envelope and $E_{k}=10^{51}$ ergs-sec ${ }^{-1}$ expanding into a $\rho=10^{-24} \mathrm{gm}-\mathrm{cm}^{-3}$ ISM (x's) is compared with the same star expanding first into a CSM that ends at $3.2 \times 10^{17} \mathrm{~cm}$ (dots).

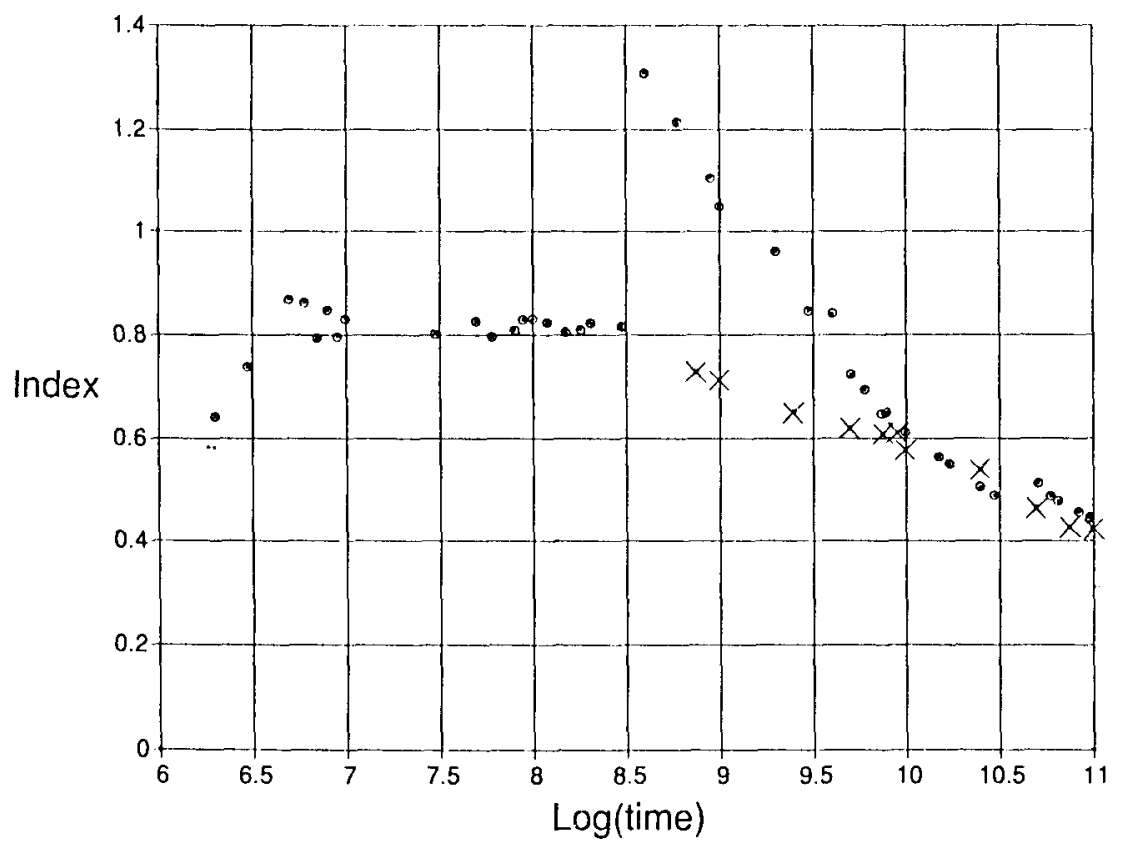

Figure 2-Effective Forward Shock Power Law Indices. The index $\eta=v_{f} t / r_{f}$ s for the two cases in Figure 1. For a blast wave $\eta=.4$. 
of the forward shock, defined as $\eta=v_{f} t / r_{f o}$, is shown on Figure 2 for both Type I cases. Four different phases can be identified in the expansion:

- First, the SN evolves according to the SSS. As can be seen in the figures, in the ISM case $r_{f s} \propto t^{4 / 7}$ until $8 \times 10^{9} \mathrm{sec}$ and in the CSM case $r_{f s} \propto t^{4 / 5}$ until $2 \times 10^{8} \mathrm{sec}$. The run was begun near the end of the SSS phase in the ISM case, and the expansion was still a little faster than predicted when the reverse shock left the envelope. We find that the internal structure of the interaction region agrees with the SSS.

- Second, the reverse shock reaches the constant density core, and the interaction region slows down. In the CSM case the forward shock reaches the end of the CSM before it slows down substantially. When the reverse shock penetrates the core, the density of the unshocked ejecta impinging on the interaction region decreases, the force of the "piston" lessens, and the expansion slows.

- Third, in the CSM case a free expansion occurs after the forward shock reaches the end of the CSM. The free expansion continues until the density in the interaction region falls to levels comparable to the ISM. During the free expansion thermal energy is reconverted to kinetic energy, and the interaction region travels at constant velocity (see Figures 1 and 2 between $8 \times 10^{8}$ sec and $2 \times 10^{10} \mathrm{sec}$ ).

- Fourth, as the reverse shock approaches the center, the drive of the "piston" become inconsequential as the unshocked ejecta falls to lower densities with smaller velocities. The remnant evolves into a blast wave. After the reverse shock hits the core, the pressure gradient causes material to rush back to the core, increasing the pressure there, and eventually reversing the inward flow. Bertschinger (1986, private communication) found in a related calculation that weak shocks bounce back and forth between the core and the contact surface, reheating the ejecta; in our calculation the ejecta are numerically noisy at this time, perhaps due to waves bouncing back and forth. While the inner region of the ejecta is complicated as the reverse shock approaches the center, and some of the resulting structure may remain long afterwards, the amount of mass involved is an insignificant fraction of the total SNR mass.

The free expansion in the CSM ends with the forward shock at approximately the same radius as the forward shock in the ISM case. At that point the ejecta have swept up approximately their own mass. Note, however, that the SNR is still expanding more rapidly than predicted by the blast wave solution $(\eta=.4)$, and even when the SN has swept up several times its own mass there remains structure inside the SNR not found in the Sedov-Taylor blast wave solution. Our results suggest that the accuracy with which the blast wave solution approximates the SNR is of the order of the ratio of swept up ISM mass to ejecta mass.

We thank E. Bertschinger, R. Chevalier, D. Cioff, C. Max and C. McKee for insightful discussions, and George Zimmerman for assistance in the use of the computer code. This work was supported by the U.S. Department of Energy, under contract No. W-7405-ENG-48 to the Lawrence Livermore National Laboratory.

Chevalier, R. 1982a, Ap. J., 258, 790 .

\section{References}

Chevalier, R. 1982b, Ap. J., 259, 302 .

Chew, G. F., Goldberger, M. L., and Low, F. E. 1956, Proc. Roy. Soc. (London), A236, 112.

Itoh, H. and Fabian, A. C. 1984, M.N.R.A.S., 208, 645.

Liang, E. P. and Chevalier, R. A. 1984, Ann. New York Acad. of Sci., 422, 233.

Weiler, K. W., Sramek, R. A., Panagia, N., van der Hulst, J. M., and Salvati, M. 1986, Ap. J., 301, 790. 